\section{e0517 FOREARM ARTERIES WITH ULTRASOUND FOR PERCUTANEOUS CORONARY PROCEDURES}

doi:10.1136/hrt.2010.208967.517

Li Yunzhi, Zhou Yujie. Department of Cardiology, Beijing Anzhen Hospital, Capital Medical University, Beijing, China

Background The radial artery has become a widely used approach for coronary angiography and intervention in patients, and the ulnar artery is another approach for elective procedure in patients.

Objective The objective of this study was to investigate the diameter, peak flow rate of blood flow and anatomy abnormalities of radial arteries and ulnar arteries of left and right forearm and to identify the influencing factors of the diameter of radial arteries and ulnar arteries in Chinese adult patients.

Methods 1112 consecutive adult patients who were to accept the selective transradial or transulnar procedures including coronary angiography and percutaneous coronary intervention were enrolled. To examine the radial arteries and ulnar arteries of left and right fore arm with colour Doppler ultrasound, to measure the diameter, peak flow rate of blood flow and anatomy abnormalities of radial arteries and ulnar arteries.

Results The diameters of right radial artery and ulnar artery were $2.316 \pm 0.507 \mathrm{~mm}$ vs $2.247 \pm 0.518 \mathrm{~mm}$ respectively; and the diameters of left radial artery and ulnar artery were $2.324 \pm 0.486 \mathrm{~mm}$ vs $2.238 \pm 0.520 \mathrm{~mm}$ respectively, there were no statistical difference ( $p>0.05)$. The diameters of radial arteries and ulnar arteries of male patients were larger than those of female patients $(p<0.05)$, but the peak flow rates of blood flow of radial artery and ulnar artery of both sides in male and female patients were similar, and there was statistical difference $(p>0.05)$. The total incidence of anatomy abnormalities of forearm arteries was $26.5 \%$. There were $91.9 \%$, $77.3 \%, 51.9 \%$ and $23.6 \%$ of total patients whose right radial artery diameter were larger and equal to the outer diameter of $5 \mathrm{Fr}, 6 \mathrm{Fr}$, $7 \mathrm{Fr}$ and $8 \mathrm{Fr}$ sheathe, respectively. Gender and arm circumference were the influencing factors of diameters of radial arteries and ulnar arteries of forearm.

Conclusions The diameters and the peak flow rates of blood flow of radial arteries and ulnar arteries of both sides were similar. The diameters of radial arteries and ulnar arteries in male patients were larger than those in female patients. The incidence of anatomy abnormalities of radial arteries were more than those of ulnar arteries. The gender and arm circumference were the influencing factors of diameters of radial arteries and ulnar arteries of forearm.

\section{e0518 A CASE REPORT OF TYPICAL ANEURYSM EXACTLY WITHIN DES IMPLANTED IN LEFT ANTERIOR DESCENDING CORONARY ARTERY}

doi:10.1136/hrt.2010.208967.518

Zheng Ying, Mao Jing-yuan, Zhang Yun, Shao Lei, Li Bin. The First Teaching Hospital of Tianjin University of Tcm

This is a case report of left anterior descending coronary artery aneurysm (CAA), typical "zig-zag" phenomenon, that developed exactly within the segment of sirolimus drug-eluting stent (DES) implanted after percutaneous coronary intervention (PCI). The patient had a history of rheumatiod arthritis. We speculated the typical coronary artery aneurysms could be related to the vascular inflammatory reaction due to both of his rheumatoid arthritis and DES implantation. For this kind of patients with the history of rheumatoid arthritis, we could get some hint, DES implantation should be careful.

\section{e0519 RAPAMYCIN SUPPRESS KRÜPPEL-LIKE FACTOR 2 EXPRESSION: MECHANISM OF ENDOTHELIAL DYSFUNCTION ASSOCIATED WITH DRUG-ELUTING STENTS}

doi:10.1136/hrt.2010.208967.519

Ma Qian, Zhou Yujie, Nie Xiaomin, Yu Miao, Gao Fei, Wang Zhijian, Nie Bin, Yan Zhenxian, Ge Hailong, Jia Dean, Yang Shiwei, Liu Xiaoli, Han Hongya, Hu Bin. Department Of Cardiology, Beijing Anzhen Hospital, Capital Medical University, Beijing, China

Objects Although rapamycin released from drug-eluting stents (DESs) affect the antithrombogenic function of endothelial cells, the exactly mechanisms underlying these effects are incompletely understood. We hypothesised that Krüppel-Like Factor 2 (KLF2), a novel and potent regulator of endothelial gene expression, might play an important role in stent thrombosis.

Methods We observed the effect of rapamycin on expression of KLF2, endothelial NO synthase (eNOS), tissue-plasminogen activator ( $t-P A)$, plasminogen activator inhibitor 1 (PAI-1) and tissue factor (TF) in Human Umbilical Vein Endothelial Cells (HUVECs). Then, with overexpression of KLF2, we mensurated the above mentioned mRNA and protein, respectively. The mRNA and protein were mensurated by RT-PCR and Western Blot Analysis. Furthermore, activation of KLF2 was evaluated by Electrophoretic Mobility Shift Assay (EMSA). Results Rapamycin made the expression and activation of KLF2 strongly reduce by $75.6 \%$ and $78.2 \%$ so as to induce long-term coronary endothelial dysfunction. In HUVECs, rapamycin made basal eNOS and t-PA decrease by $80 \%$ and $87.8 \%$, while making basal PAI-1 and TF increase by 2.5 and 1.5-fold. Overexpression of KLF2 strongly induced eNOS and t-PA expression and reduced PAI-1 and TF expression, reversing protein above-mentioned near to normal state.

Conclusions The data indicated that rapamycin strongly inhibited the expression of KLF2, meanwhile, reduced anticoagulants (eNOS and t-PA) and induced procoagulants (PAI-1 and TF). KLF2 played an important role in stent thrombosis owing to rapamycin-induced endothelial dysfunction, which might be a part of mechanism of stent thrombosis associated with DESs.

\section{e0520 STATIN EFFECTS IN STENT THROMBOSIS INDUCED BY RAPAMYCIN RELEASING FROM DRUG-ELUTING STENTS THROUGH KRÜPPEL-LIKE FACTOR 2 OVEREXPRESSION}

doi:10.1136/hrt.2010.208967.520

Ma Qian, Zhou Yujie, Nie Xiaomin, Yu Miao, Gao Fei, Wang Zhijian, Nie Bin, Yan Zhenxian, Ge Hailong, Jia Dean, Yang Shiwei, Liu Xiaoli, Han Hongya, Hu Bin. Department of Cardiology, Beijing Anzhen Hospital, Capital Medical University, Beijing, China

Objects As we studied before, rapamycin released from drug-eluting stents (DESs) affected the antithrombogenic function of endothelial cells through Krüppel-Like Factor 2 (KLF2) decrease. However, 3-hydroxy-3-methylglutaryl coenzyme A reductase inhibitors (statins) are known to modulate endothelial function by inducing KLF2. Here we report that statin-induced expression of KLF2 can reverse stent thrombosis.

Methods We observed the effect of rapamycin on expression of KLF2, endothelial NO synthase (eNOS), tissue-plasminogen activator (t-PA), plasminogen activator inhibitor 1 (PAI-1) and tissue factor (TF) in Human Umbilical Vein Endothelial Cells (HUVECs). And then KLF2 mRNA was induced by treatment with multiple statins in a concentration-dependent manner. The mRNA and protein were mensurated by RT-PCR and Western Blot Analysis Furthermore, activation of KLF2 was evaluated by Electrophoretic Mobility Shift Assay (EMSA). 